\title{
XXXIII. Logarithmic lazytongs and lattice-works
}

\section{Thomas H. Blakesley}

To cite this article: Thomas H. Blakesley (1907) XXXIII. Logarithmic lazytongs and lattice-works , Philosophical Magazine Series 6, 14:81, 377-381, DOI: 10.1080/14786440709463693

To link to this article: http://dx.doi.org/10.1080/14786440709463693

$$
\text { 册 Published online: } 16 \text { Apr } 2009 .
$$

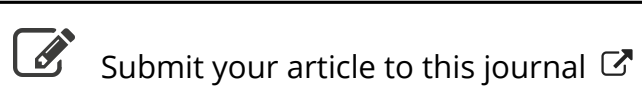

\footnotetext{
Џll Article views: 2
}

Q View related articles $\square$

7 Citing articles: 1 View citing articles $\square$ 
and to the first order of $b / a$,

$$
\begin{gathered}
\psi=-\frac{2}{3 \pi} \frac{k^{2} a^{3}}{r}\left(1-\frac{7 k^{2} a^{2}}{25}+\frac{k^{2} n^{2}}{10} \cos ^{2} \theta\right) \cos \theta e^{-2 k r} \\
-\frac{k^{2} a^{2} b}{3 r}\left(1+\frac{8 \cos \theta}{\pi^{2}}\right) e^{-t k^{r} r}+\frac{k^{4} a^{4} b}{r}\left(-\frac{2}{45}+\frac{76}{75} \frac{\cos \theta}{5 \pi^{2}}-\frac{1}{90} \cos ^{2} \theta-\frac{4}{3 \pi^{2}} \cos ^{3} \theta\right) e^{-2 i r}
\end{gathered}
$$

When the incidence is oblique the analysis is much more cumbrous, and there is little interest in carrying it beyond the approximations given by Lord Rayleigh in the paper cited above.

XXXIII. Logarithmic Lazytongs and Lattice-works. By Thomas H. Blakesley *

$\checkmark$ THE point of view in which the Equiangular Spiral is 1 usually regarded is that implied in its name, viz., the curve which makes the same angle with its radius vector,

$$
r \frac{d \theta}{d r}=\tan \alpha .
$$

It is rather from what I may perhaps call its polygonal character that I shall present and apply it in this paper. By this I mean that it is a circumscribing curve to polygonal figures following simple laws.

If a series of equal straight lines form a consecutive number of the sides of a regular polygon, the circumscribing circle is absolutely determined.

But if those straight lines, still maintaining the equality of the angles between any consecutive two, in magnitude form a geometrical series, the circumscribing curve will be the equiangular spiral.

According to the value of the angle between consecutive lines, one may speak of the figure as a regular logarithmic pentagon, hexagon, octagon, \&c., and more generally as a regular logarithmic polygon. The regularity consists in the equality of the angles between the lines, and in those subtended by them at the pole of the spiral.

Consecutive chords are those straight lines which form consecutive sides of a logarithmic polygon.

* Communicated by the Physical Society : read March 22, 1907. 
Some geometrical matters more immediately arising from this view of the curve may be introduced.

The problem of finding the pole when two consecutive chords are given is solved thus:-Let $A B, B($, be the consecutive chords given. Complete the parallelogram, and let $\mathrm{BD}$ be the diagonal throngh $\mathrm{B}$. Make the angle BCP

Fig. 1.

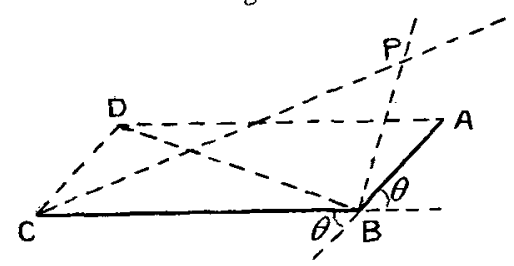

equal to the angle $\mathrm{DBC}$, and make the angle $\mathrm{ABP}$ equal to the same angle, Then $\mathrm{P}$ is the pole of the spiral in which $\mathrm{AB}, \mathrm{BC}$ are consecutive chords.

As an alternative to the setting off of one of the angles $B C P$ or $A B P$, the angle $B A P$ may be made equal to $D B A$.

$\mathrm{Or}$, as it may be shown that the product of $\mathrm{PB} . \mathrm{DB}$ is equal to that of $\mathrm{AB} . \mathrm{BC}$ :

$B P$ may be easily calculated from the data, viz., the values of $\mathrm{AB}$ and $\mathrm{BC}$, and the angle between them.

If one of the two chords (say $\mathrm{BC}$ ) is maintained in position, but the other $\mathrm{BA}$ is made to turn round $\mathrm{B}$, so as to vary the angle between the chords, the pole $\mathrm{P}$ will describe a circle whose centre is in the line CB, produced if required

If $\theta$ is the angle between the two consecutive chords externally, the characteristic angle of the spiral $(a)$ will be given by

$$
\tan \alpha=\frac{\theta}{\log \frac{\mathrm{BC}}{\mathrm{AB}}} .
$$

In any cases therefore in which $\frac{\theta}{\log \frac{\mathrm{BC}}{\mathrm{AB}}}$ has the same
alue, the spirals are similar.

It follows that, in any mechanical construction of linkages \&c., if we can keep $\frac{\mathrm{BC}}{\mathrm{AB}}$ constant, but can at the same time cause $\theta$ to vary, we have the power of changing $\alpha$, that is to say the one thing which settles the character of the equiangular spiral. 
If now two straight rods or lines, $\mathrm{AB}, \mathrm{CD}$ (fig. 2) are taken in one plane, and meeting in $\mathrm{E}$ (whether in their actual lengths as shown, or in their geometrical productions, is immaterial), and so conditioned that $\mathrm{A}, \mathrm{C}, \mathrm{B}, \mathrm{D}$ lie in one circle, then the products of their segments are equal or $\mathrm{CE} . \mathrm{ED}=\mathrm{AE} . \mathrm{EB}$.

Fig. 2.

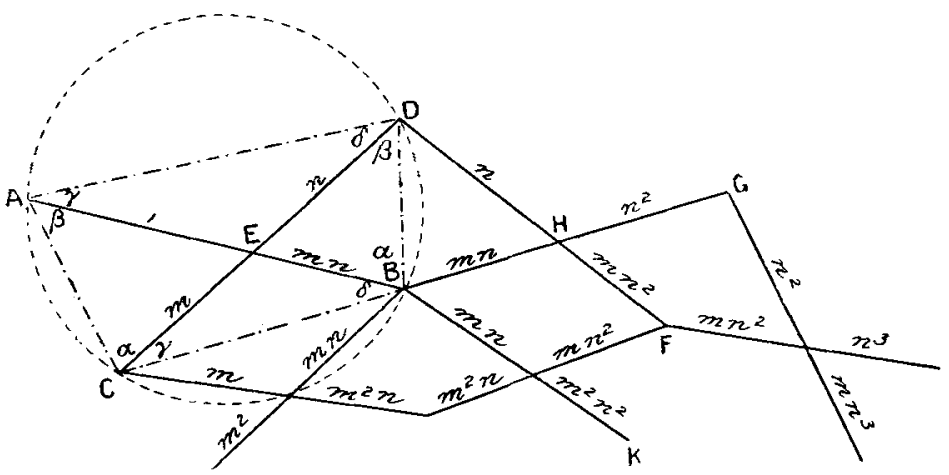

It follows that $E$ remaining the same for both rods A, C, B, D will always lie on a circle, whatever be the angle between the rods.

If $\mathrm{DE}=n . \mathrm{AE}$, and $C \mathrm{E}=m . \mathrm{AE}$, then the condition is fulfilled if $\mathrm{EB}=m n . \mathrm{AE}$; $n$ and $m$ may have any values whatever.

It will be convenient for geometrical reasoning to imagine or describe the straight lines $\mathrm{AC}, \mathrm{CB}, \mathrm{BD}, \mathrm{DA}$. Then the triangles $\mathrm{DEB}, \mathrm{AEC}$, are similar, and

$\mathrm{DE}: \mathrm{EB}: \mathrm{BD}:: \mathrm{AE}: \mathrm{EC}: \mathrm{CA}:: n: 1$.

Similarly regarding the triangles $\mathrm{CEB}, \mathrm{AED}$, they are similar, and $\mathrm{CE}: \mathrm{EB}: \mathrm{BC}:: \mathrm{AE}: \mathrm{ED} ; \mathrm{DA}:: m: 1$.

Call the angles ECA, EAC, EAD, EDA, $\alpha, \beta, \gamma$, and $\delta$ respectively. Then also the angles $\mathrm{EBD}, \mathrm{EDB}, \mathrm{ECB}, \mathrm{EBC}$ are $x, \beta, \gamma$, and $\delta$ respectively.

Now DB may be derived from $\mathrm{AC}$, as regards direction and magnitude, by allowing $\mathrm{AC}$ to revolve first through $\mathrm{ACE}$ or $\alpha$ in one direction, and then through $\mathrm{EDB}$, or $\beta$ in the opposite direction, and by reduction in the ratio $1: n$.

Thus $\mathrm{DB}$ makes with $\mathrm{AC}$ the angle $\alpha-\beta$, and $\mathrm{DB}=n$. $\mathrm{AC}$.

Similarly $C B$ makes with AD the angle $\overline{\gamma-\delta}$ and $\mathrm{CB}=m . \mathrm{AD}$.

Now suppose another pair of rods DF, BG jointed at $\mathrm{H}$, and similar to the first pair in all respects but bearing the ratio to them of $n: 1$, jointed on to the first pair at $B$ and $D$. 
All the lines in this, which may be called the second cell in the direction $n$, including those of the circumscribing circle are homologous in a ratio $n: 1$ with the corresponding lines of the first cell, and the angular displacement relatively to the first cell is $\overline{\alpha-\beta}$.

To the points FG may now be connected a third cell, constituted in a similar manner to the second, and so on indefinitely.

To AC may be connected a similar cell bearing to the first cell the linear proportion of $1: n$ and to this another, and so on indefinitely in the direction $\frac{1}{n}$.

Such a line of cells may be called a logarithmic lazytongs.

It is clear that all such points as $A, D, G$ lie upon an equiangular spiral, the tangent of whose characteristic angle is equal to $\frac{\overline{\alpha-\beta}}{\log n}$.

As all the cells of a series are similar, any motion involving the increase or decrease of the angle between the bars of one cell, will be accompanied by the same change in angle in all the cells.

The sides AD, CB may also have cells attached to them, the same rules as before being observed. $m$ will take the place of $n$ in the change of scale and $\overline{\gamma-\delta}$ the place of $\overline{\alpha-\beta}$ in the change of direction.

It is to be pointed out that if two cells in the $m$ direction be applied to $\mathrm{BC}$ and $\mathrm{BF}$, the other adjacent points of the two cells will coincide. In other words, the cell on BF may be considered as derived from the first cell either by one move in the $n$ direction followed by another in the $m$ direction, or by one in the $m$ direction followed by a second in the $n$ direction. Whence it follows that the whole of a plane surface may be occupied by a plenum of cells forming an infinite lattice-wurk, in which, if the angle between the crossbars of any one cell is changed, an equal change takes place in that between the cross-bars of any other cell.

In the $m$ direction the tangent of the angle characterisqic of the spiral through such points as $A$ and $C$ is equal to $\overline{\frac{\gamma-\delta}{\log _{\ell} m}}$.

There is a common pole for both the $m$ and the $n$ spirals.

If for the sake of easy description we liken the plenum of cells so obtained to a chessboard, the rooks' moves would 
take place either in the $n$ direction or in the $m$ direction, or in the $\frac{1}{n}$ direction or in the $\frac{1}{m}$ direction.

But the bars $A B$ \&c. which lie in what would then be called a bishop's move, would also have their extremities in an equiangular spiral having the same pole as the other sets of spirals. The angle between successive chords (external) will be in this case $\overline{\alpha-\beta}+\overline{\gamma-\delta}$, and the change ratio of the chords $m n$, so that the angle characteristic of the spiral will have for its tangent

$$
\frac{\overline{\alpha-\beta}+\overline{\gamma-\delta}}{\log _{e} m n} \text {. }
$$

Circles and straight lines are only limiting cases of equiangular spirals. They may therefore be awaited among the cases arising from changing $m, n$ or the angle between the cross-bars.

If $n=1$ the $n$ spirals become circles, and since in that case $\gamma=\delta$, the $m$ spirals become straight lines.

If $m=1$ the $m$ spirals become circles and the $n$ spirals straight lines.

If both $m$ and $n$ are equal to unity, both systems are straight lines.

If $m n=1$, in which case $A B$ is bisected in $E$, there is no change of scale along the bishop's move in the direction $\mathrm{AB}$. In consequence the spirals through $\mathrm{AB}$ are circles.

Similarly if $m=n$ CD is bisected, and such lines lie on circles.

Such lines as $\mathrm{AB}, \mathrm{BK}$, \&c. may under some circumstances lie in a straight line.

The displacement in angle of such lines is $(\alpha-\beta+\gamma-\delta)$.

If this is equal to zero

$$
\begin{aligned}
& \alpha+\gamma=\bar{\beta}+\bar{\delta} \\
\therefore & \beta+\bar{\delta}=\frac{\pi}{2} .
\end{aligned}
$$

Thus the circumscribing circle must have $\mathrm{AB}$ for a diameter. Therefore as $\mathrm{D}$ and $\mathrm{C}$ are both on that circle (DD must either be equal to $\mathrm{AB}$, in which case the two chords bisect each other $(m=n=1)$, or $\mathrm{CD}$ is less than $\mathrm{AB}$. It is therefore only the longer of the cross-bars which, by the variation of the angle between them, can come into a straight line. The longer cross-bar is also that one which is divided most unequally, since the product of the segments is the same in the two bars.

Phil. May. S. 6. Vol. 14. No. 81. Sept. 1907. 2 D 